Abstract P1-S1.30 Table 1 Chlamydia trachomatis prevalence, detected by culture and RT-PCR tests, among male attendees of the urologist's office in St. Petersburg

\begin{tabular}{lllll}
\hline \multirow{2}{*}{ No men tested } & & \multicolumn{3}{c}{ Chlamydia trachomatis } \\
\cline { 3 - 5 } & & CT - & CT + & CT prevalence \\
\hline In total & 907 & 849 & 58 & $6.4 \%$ \\
Culture test, only & 466 & 456 & 10 & $2.2 \%$ \\
RT-PCR test, only & 804 & 747 & 57 & $7.6 \%$ \\
Culture and RT-PCR, both & 376 & 351 & 25 & $6.7 \%$ \\
\hline
\end{tabular}

Conclusions Our study showed that-(1) CT prevalence among the Russian population is still high especially in MSM. (2) Risk factor include-being symptomatic at the time of testing $(p=0.0043)$, inconsistent condom use and practicing sex with men (both$\mathrm{p}<0.0001$ ). (3) All samples found culture and/or RT-PCR CT + in Russia were confirmed CT + using molecular biological techniques in Amsterdam, showing the validity of CT detection in this study in St. Petersburg. At the moment we additionally-A) extent.

\section{P1-S1.31 CHLAMYDIA TRACHOMATIS IN NORWAY, 1988-2009}

doi:10.1136/sextrans-2011-050108.31

F E Skjeldestad, H Kløvstad. Norwegian Institute of Public Health, Oslo, Norway

Background Chlamydia trachomatis is the most commonly reported STI in Europe. We present the epidemiology of $C$ trachomatis in Norway from 1988 to 2009

Methods Until 2004 the Norwegian Surveillance system of infectious diseases (MSIS) captured the total number of diagnosed cases and tests performed from all diagnosing laboratories. From 2005 date of diagnosis, birth year, sex and municipality of living has been included for each diagnosed case. We calculated testing rates, incidence rates (IR) and incidence rate ratios (IRR) with 95\% CIs.

Results The IR of diagnosed cases was at its lowest in 1995 (287/ $100000)$ and increased yearly until 2008 (467/100 000). The testing rate reached a peak in 1991 (8000/100 000), decreased towards year 2000 (5300/100 000) and has been stable until 2009 (5500/100 000). The proportion positive tests has increased yearly from $4.1 \%$ in 1993 to $8.6 \%$ in 2009. The highest mean annual IR was found in the age group 20-24 years for both men (2983/100 000) and women (4818/100 000). Women were 1.6 times more likely (IRR of $1.6,95 \%$ CI 1.6 to 1.62 ) to be diagnosed with $C$ trachomatis compared to men, and inhabitants in the two most northern regions were more likely to be diagnosed than the rest of the country (IRR $1.6,95 \%$ CI 1.5 to 1.7).

Conclusion The IR of C trachomatis in Norway is increasing while the testing rate is stable. More detailed data of the case mix among the tested subjects is needed to interpret the observed trends.

\section{P1-S1.32 UROGENITAL CHLAMYDIA TRACHOMATIS IS A HYPERENDEMIC DISEASE IN PARAMARIBO SURINAME. RESULTS FROM A MULTIETHNIC SOCIETY}

\section{doi:10.1136/sextrans-2011-050108.32}

${ }^{1} \mathrm{~J}$ van der Helm, ${ }^{2} \mathrm{~A}$ Grunberg, ${ }^{1} \mathrm{~A}$ Speksnijder, ${ }^{3,4} \mathrm{H}$ de Vries, ${ }^{5} \mathrm{~L}$ Sabajo. ${ }^{1}$ Health Service Amsterdam, Amsterdam, Netherlands; ${ }^{2}$ Lobi Foundation, Suriname; ${ }^{3}$ Health Service Amsterdam, Academic Medical Center, University of Amsterdam, Netherlands; ${ }^{4}$ Centre for Infections and Immunity Amsterdam CINIMA, Netherlands; ${ }^{5}$ Dermatology Service, Ministry of Health, Suriname

Background Suriname is a multicultural society with many ethnic groups, such as Creoles and Maroons (both descendants from the
African diaspora due to slave trade), Indian, Javanese, and Chinese (all descendants from labour immigrants), Caucasians (descendants from Dutch farmers) indigenous Amerindian people and Mixed race persons. The prevalence of Chlamydia trachomatis (Ct) among Surinamese living in the Netherlands visiting the Amsterdam STI clinic is high, up to $17 \%$. However, due to lack of testing facilities the prevalence of $\mathrm{Ct}$ in Suriname is unknown. We aimed to estimate the prevalence and risk factors for $\mathrm{Ct}$ in two outpatient clinics in Paramaribo, Suriname, S.A.

Methods Between March 2008 and February 2010436 men and 292 women were included in a high risk STI clinic and 829 women were included in a low risk birth control outpatient clinic in Paramaribo, Suriname. Male urine samples and nurse collected vaginal swabs were obtained for nucleic acid amplification test (NAAT) (APTIMA Combo 2, Gen-Probe, San Diego, USA) testing in Amsterdam. Logistic regression analysis was used to identify risk factors for $\mathrm{Ct}$ infection.

Results Ct prevalence was 23\% for men and 20\% for women at the high risk STI clinic and 10\% for women at the low risk birth control clinic. The distribution of individuals included from each ethnic group is representative for the ethnic composition of the Surinamese population. One of the more striking outcomes was that a high $\mathrm{Ct}$ prevalence was found among Creoles (20\%), Mixed race (17\%) and Javanese (16\%) individuals. Adjusted for age and test site, the Ct prevalence was significantly higher among Creole and Javanese individuals compared to Indian, OR 2.0 (95\% CI 1.2 to 3.3) and 2.1 (95\% CI 1.2 to 3.8 ) respectively (Abstract P1-S1.32 table 1). Young age ( $\leq 24$ years) was significantly associated with $\mathrm{Ct}$ infection OR 3.1 (95\% CI 2.1 to 4.7). Educational level was not associated with Ct infection.

Abstract P1-S1.32 Table 1 Univariate and multivariate logistic regression analyses of factors associated with $\mathrm{Ct}$ infection

\begin{tabular}{lcll}
\hline & $\begin{array}{l}\text { NAAT } \\
\text { positive (\%) }\end{array}$ & $\begin{array}{l}\text { Univariate OR } \\
\text { (95\% Cl) }\end{array}$ & $\begin{array}{l}\text { Multivariate adjusted } \\
\text { OR (95\% Cl)* }\end{array}$ \\
\hline $\begin{array}{l}\text { Gender } \\
\text { Male }\end{array}$ & $100 / 436(22.9)$ & $2.1(1.6$ to 2.8$)$ & - \\
$\quad$ Female & $138 / 1120(12.3)$ & 1 & - \\
Test site & & & \\
$\quad$ STI clinic (high-risk) & $157 / 728(21.6)$ & $2.5(1.9$ to 3.4$)$ & $2.4(1.8$ to 3.4$)$ \\
$\quad$ Birth c. clinic (low-risk) & $81 / 828(9.8)$ & 1 & 1 \\
Age (years) & & & \\
$\quad$ 24 & $104 / 438(23.7)$ & $3.5(2.3$ to 5.2$)$ & $3.1(2.1$ to 4.7$)$ \\
$25-34$ & $96 / 664(14.5)$ & $1.9(1.3$ to 2.8$)$ & $1.9(1.2$ to 2.8$)$ \\
$\geq 35$ & $37 / 453(8.2)$ & 1 & 1 \\
Ethnic group & & & 1 \\
$\quad$ Indian & $23 / 291(7.9)$ & 1 & \\
$\quad$ Creole & $93 / 459(20.3)$ & $3.0(1.8$ to 4.8$)$ & $2.0(1.2$ to 3.3$)$ \\
Javanese & $28 / 180(15.6)$ & $2.1(1.2$ to 3.9$)$ & $2.1(1.2$ to 3.8$)$ \\
Maroon & $35 / 261(13.4)$ & $1.8(1.0$ to 3.1$)$ & $1.1(0.6$ to 1.9$)$ \\
$\quad$ Mixed race & $50 / 292(17.1)$ & $2.4(1.4$ to 4.1$)$ & $1.5(0.9$ to 2.6$)$ \\
Indigenous Amerindian & $3 / 25(12.0)$ & $1.6(0.4$ to 5.7$)$ & $1.0(0.3$ to 3.8$)$ \\
Chinese & $0 / 14(0)$ & 0 & 0 \\
Caucasian & $4 / 19(21.1)$ & $3.1(0.9$ to 10.1$)$ & $1.7(0.5$ to 5.6$)$ \\
\hline
\end{tabular}

${ }^{*}$ ORs in the multivariate model are adjusted for all factors for which adjusted ORs are shown.

Conclusion This is the first report on Ct epidemiology in Suriname. With a prevalence of $10 \%$ in the low risk birth control clinic population and $23 \%$ in the high risk STI clinic, the situation is alarmingly high and testing facilities are urgently needed to minimise further spread and disease burden of $\mathrm{Ct}$. Moreover, the $\mathrm{Ct}$ prevalence is not equally distributed among the various ethnic groups. Especially the Creole and Javanese Surinamese populations are disproportionally affected. 